Cahiers de la recherche sur les droits

Cahiers

Fur les Droits fondamentaux

3 | 2004

Surveiller et punir / Surveiller ou punir ?

\title{
La situation des établissements pénitentiaires de Basse-Normandie
}

Jean-Louis Touzé et Céline Valenza

\section{OpenEdition}

Édition électronique

URL : https://journals.openedition.org/crdf/7441

DOI : $10.4000 /$ crdf.7441

ISSN : 2264-1246

Éditeur

Presses universitaires de Caen

Édition imprimée

Date de publication : 1 octobre 2004

Pagination : 15-22

ISBN : 2-84133-221-7

ISSN : $1634-8842$

Référence électronique

Jean-Louis Touzé et Céline Valenza, « La situation des établissements pénitentiaires de Basse-

Normandie », Cahiers de la recherche sur les droits fondamentaux [En ligne], $3 \mid 2004$, mis en ligne le 18 décembre 2020, consulté le 14 novembre 2022. URL : http://journals.openedition.org/crdf/7441; DOI https://doi.org/10.4000/crdf.7441

Tous droits réservés 


\title{
La situation des établissements pénitentiaires de Basse-Normandie
}

\author{
Jean-Louis TOUZÉ \\ Membre du Conseil économique et social régional de Basse-Normandie \\ Céline VALENZA \\ Chargée de mission auprès du Conseil économique et social régional de Basse-Normandie
}

I. Objectifs et orientations de l'étude relative au milieu carcéral en Basse-Normandie

II. Les structures carcérales, les publics détenus et les dispositifs d'insertion au sein des détentions bas-normandes
A. L'institution pénitentiaire en Basse-Normandie
B. Structures carcérales et populations incarcérées en Basse-Normandie
C. Les dispositifs d'insertion sociale et professionnelle au sein des détentions bas-normandes

III. Propositions émises par le Conseil économique et social régional

\section{Objectifs et orientations de l'étude relative au milieu carcéral en Basse-Normandie}

L'intérêt porté par le Conseil économique et social régional (CESR) à l'univers carcéral en Basse-Normandie, notamment pour appréhender les dispositifs d'insertion sociale et professionnelle destinés aux personnes détenues, tient à une double interrogation et à un constat.

La machine carcérale, en menant à l'oisiveté et à l'ennui les publics qu'elle détient, ne possède-t-elle pas un effet socialement déstructurant?

À l'inverse, ne favorise-t-elle pas la réintégration sociale et professionnelle en venant en aide à ceux qui désirent s'amender?

Quoi qu'il en soit, il convient d'avoir à l'esprit que les publics concernés, hommes, femmes, adultes et mineurs, représentent, avant et après incarcération, des populations locales qui, certainement plus que d'autres, méritent une prise en charge par les pouvoirs publics, le secteur privé et le tissu associatif de proximité.

De ce fait, les différents acteurs locaux sont légitimement enclins à s'interroger sur la nature, le degré de développement et la portée des dispositifs de réintégration socioprofessionnelle déclinés au sein de chacune des six structures carcérales bas-normandes.

Dans le sens même de cette préoccupation et pour comprendre au mieux le contexte régional, le CESR a souhaité, dans son étude, analyser tout d'abord les caractéristiques générales du système carcéral français.

Ainsi, après un éclairage sur l'histoire de la peine privative de liberté et sur la genèse de la prison républicaine, le document évoque largement l'organisation structurelle et territoriale de l'institution pénitentiaire contemporaine.

L'attention est ensuite portée aux problématiques et aux enjeux que pose, aujourd'hui en France, la prise en charge des personnes détenues.

Différentes questions sont développées dans ce cadre: 
- l'évolution et les profils actuels des publics incarcérés ;

- les lieux et conditions de vie en détention d'une population pénale complexe et hétérogène ;

- l'implication délicate des acteurs du milieu carcéral;

- le contrôle et l'évaluation des actions menées au sein des détentions.

Le contexte national rigoureusement posé, le CESR consacre la deuxième partie de son travail à l'institution pénitentiaire dans la région, et aux dispositifs d'insertion sociale et professionnelle mis en œuvre dans chacune des structures carcérales bas-normandes.

\section{Les structures carcérales, les publics détenus et les dispositifs d'insertion au sein des détentions bas-normandes}

\section{A. L'institution pénitentiaire en Basse-Normandie}

L'institution pénitentiaire est présente sur le territoire bas-normand à différents niveaux et à travers différentes structures.

Tout d'abord, la Basse-Normandie se trouve administrativement et hiérarchiquement liée à la Région pénitentiaire de l'Ouest dont l'aire d'influence s'étend également aux régions Bretagne et Pays-de-la-Loire, et dont le siège se situe à Rennes.

D'autres services de l'Administration pénitentiaire se déclinent par ailleurs à un échelon plus déconcentré sur le territoire bas-normand; il s'agit des trois services pénitentiaires d'insertion et de probation à vocation départementale, d'une part, et des six structures carcérales, d'autre part.

\section{B. Structures carcérales et populations incarcérées en Basse-Normandie}

Les " prisons bas-normandes » totalisent 1482 places d'hébergement théoriques et se répartissent en quatre maisons d'arrêt et deux établissements pour peines.

\section{Les maisons d'arrêt et leur population pénale}

Localisées à Alençon, Caen, Cherbourg et Coutances, les maisons d'arrêt disposent ensemble de 445 places d'hébergement théoriques.

Contrairement aux maisons d'arrêt de Coutances et de Cherbourg, construites dans les années 1820, et à la maison d'arrêt de Caen érigée à partir de 1899, le Château des ducs d'Alençon constitue un édifice médiéval du $\mathrm{XII}^{\mathrm{e}}$ siècle qui n'a jamais eu pour vocation principale de devenir un lieu d'emprisonnement.

Aujourd'hui habilitées à recevoir des prévenus et des condamnés, les maisons d'arrêt de la région connaissent un surencombrement fréquent, voire continu, de leur «quartier hommes» respectif.
Ainsi, le 20 janvier dernier, la maison d'arrêt de Caen détenait 432 hommes majeurs pour 222 places, affichant un taux d'occupation de ce quartier de détention de $194 \%$.

À cette même date, le taux d'encombrement des « quartiers hommes », n'incluant pas les condamnés placés en semi-liberté, était de $182 \%$ à Alençon, de $179 \%$ à Coutances et de $171 \%$ à Cherbourg.

Les maisons d'arrêt de la région accueillent donc principalement des hommes majeurs auxquels s'ajoutent une trentaine de femmes et une dizaine de mineurs, au sein de quartiers spécifiques de la maison d'arrêt de Caen.

Jusqu'à l'été dernier, quelques femmes étaient également hébergées à Coutances dans un lieu réservé de la détention, lequel reçoit aujourd'hui des hommes placés en semi-liberté.

80 à $90 \%$ des publics détenus derrière les murs de ces quatre structures carcérales sont domiciliés en BasseNormandie.

Enfin, près de la moitié des détenus à la charge des maisons d'arrêt au cours de l'année 2002 se sont trouvés incarcérés pour des faits de vols et / ou de coups et blessures volontaires.

\section{Les établissements pour peines et leurs publics détenus}

À côté des maisons d'arrêt, notre région compte deux établissements pour peines que sont le centre pénitentiaire de Caen et le centre de détention d'Argentan.

Le premier témoigne à travers l'un de ses bâtiments de son passé de maison de lépreux et offre, par le reste de son architecture, la vision d'une structure datant des années 1950, puis 1990 avec la construction d'un bâtiment médical moderne.

Le second fait partie du programme " 13000 places », initié en 1988, et a ouvert ses portes en 1991. Reflet de l'architecture carcérale contemporaine, il fonctionne en gestion mixte (publique / privée).

Tous deux hébergent exclusivement des hommes majeurs, condamnés de manière définitive; quelques mineurs et prévenus sont toutefois accueillis en hospitalisation au service médico-psychologique régional implanté au centre pénitentiaire de Caen.

Les deux structures pénitentiaires disposent comme tout établissement pour peines d'une stricte limitation du nombre de leurs places d'hébergement, atteignant respectivement les chiffres de 599 à Argentan et 438 à Caen.

Le taux d'encombrement était ainsi de $98 \%$ à Argentan et de $94 \%$ à Caen (non inclus les détenus du SMPR), le 20 janvier de cette année.

Contrairement aux publics majoritairement locaux des maisons d'arrêt, les condamnés du centre de détention d'Argentan proviennent essentiellement des régions limitrophes de la Basse-Normandie.

Au regard de caractéristiques pénales observées sur l'année 2002, 32,7\% d'entre eux purgeaient une peine pour viol; $28,5 \%$ étaient incarcérés pour vol et $17,5 \%$ avaient commis une infraction à la législation sur les stupéfiants. 
Le centre pénitentiaire de Caen reçoit quant à lui une population pénale, issue de la France entière, composée d'une majorité d'auteurs de délits et crimes à caractère sexuel (près de $78 \%$ en 2002).

\section{Les dispositifs d'insertion sociale et professionnelle au sein des détentions bas-normandes}

Destinée à orienter les criminels et les délinquants vers les voies de l'amendement, la prison se veut également un lieu d'insertion sociale et professionnelle pour les publics qu'elle retient.

Pour cela, elle a recours à un panel de dispositifs complémentaires visant à favoriser l'intégration sociale, l'insertion professionnelle, l'orientation et la préparation du retour à la vie libre.

Le développement de ces mesures demeure néanmoins fortement tributaire, en Basse-Normandie comme ailleurs, des possibilités offertes en termes d'espace par les différentes architectures carcérales.

\section{Les actions en faveur de l'intégration sociale}

a. Le maintien des liens familiaux

Il s'opère à travers les différents modes et moyens de communication que sont les relations épistolaires et téléphoniques, les rencontres avec les proches à l'occasion des «parloirs-familles» et lors des éventuelles permissions de sortir octroyées aux condamnés.

Toutes les personnes incarcérées dans la région peuvent envoyer et recevoir du courrier, dans le respect de la réglementation en vigueur.

En revanche, seuls les condamnés du centre de détention d'Argentan et du centre pénitentiaire de Caen ont accès aux échanges téléphoniques, cette possibilité n'étant pas offerte aux publics des maisons d'arrêt.

Les conditions d'accueil des familles et de rencontre des détenus à l'occasion des parloirs different fortement sur le territoire régional.

Ainsi, aucun accueil spécifique des familles n'est mis en œuvre aux maisons d'arrêt de Cherbourg et d'Alençon, où aucun local même ne permet aux visiteurs de patienter jusqu'au début du parloir.

Une salle d'attente est, en revanche, mise à disposition des familles au centre pénitentiaire de Caen sans dispositif d'accueil particulier, néanmoins.

Enfin, un lieu et un accompagnement spécifiques, faisant intervenir des associations, existent sur les sites des maisons d'arrêt de Caen et de Coutances, ainsi qu'à Argentan. Il importe de préciser toutefois qu'aucun transport public ne relie le centre de détention au centre-ville d'Argentan situé à 5 kilomètres.

Au sein des quatre maisons d'arrêt, le planning des parloirs s'organise au regard de la situation pénale des détenus (prévenus / condamnés), durant la semaine.

$\mathrm{Au}$ centre de détention d'Argentan, les visites se déroulent au cours du week-end et des jours fériés, tandis qu'elles sont quotidiennes et non limitées en nombre, du lundi au samedi, au centre pénitentiaire de Caen.
Hormis à Argentan, les «parloirs-familles» se tiennent au sein de locaux collectifs impliquant une promiscuité visuelle et phonique inévitable entre les détenus, leurs codétenus et les différents visiteurs.

Enfin, il importe de souligner que l'octroi d'une permission de sortir en vue du maintien des liens familiaux accordée à certains condamnés, notamment au sein des deux établissements pour peines, peut s'avérer délicat lorsque ceux-ci n'ont pas d'hébergement à l'extérieur ou sont dans l'incapacité de pourvoir aux dépenses induites par la sortie.

\section{b. L'assistance spirituelle et l'implication du monde associatif et des acteurs locaux}

Le culte catholique et le culte protestant sont tous deux régulièrement représentés au sein des six structures carcérales de la région.

Des contacts plus ponctuels sont recherchés auprès d'autorités religieuses d'autres confessions lorsque la demande émane d'un ou plusieurs détenus.

Les fêtes religieuses les plus importantes sont observées.

Par ailleurs, de nombreuses associations et différents acteurs locaux interviennent dans des domaines variés au sein des établissements pénitentiaires de BasseNormandie :

- Accueil des familles (Arc en Ciel à la maison d'arrêt de Caen, La Cordée à Argentan);

- Accompagnement individuel des détenus (membres de l'ANVP);

- Activités socio-éducatives (troupe de théâtre ACTÉA à la maison d'arrêt de Caen, Comité coutançais d'action culturelle);

- Éducation à la santé (Centre départemental de prévention de l'alcoolisme à la maison d'arrêt de Caen, les Alcooliques anonymes au centre pénitentiaire, Drog'Aide 61 à Alençon);

- Enseignement (Étudiants du Génépi à Caen, «La maison des mots » à Argentan);

- Travail pénitentiaire (ARTEC, AFPCM au centre pénitentiaire de Caen, bibliothèque municipale de Cherbourg-Octeville).

\section{c. La lutte contre les situations d'indigence}

L'Administration pénitentiaire s'attache à mener depuis peu des actions de prévention et de lutte contre l'indigence par un repérage et une intervention ciblés en direction des publics pécuniairement nécessiteux.

Cette démarche repose sur la réunion de commissions pluridisciplinaires à Coutances, Cherbourg et Argentan, associant les acteurs principaux de chaque site : direction, SPIP, responsables de la comptabilité ou de l'économat, représentants ici ou là de l'enseignement, de la formation professionnelle, du service médical, de l'association socioculturelle de l'établissement, d'associations caritatives, etc.

Aucune commission de ce type n'existait, en revanche, au moment de l'élaboration de l'étude, à Alençon et au sein des deux établissements du Calvados, dans lesquels néanmoins la démarche était en phase de structuration. 
Il importe de voir toutefois que les réponses apportées aux situations d'indigence diffèrent grandement d'un lieu carcéral à l'autre.

L'indigence est reconnue en deçà de 25 euros sur le pécule disponible à Cherbourg, la différence pour atteindre cette somme étant gratuitement allouée par l'association socioculturelle de l'établissement.

À Coutances, l'état d'indigence est reconnu en deçà de 45 euros mais ne conduit à aucun versement.

Sur les deux sites, les détenus peuvent bénéficier de la distribution gratuite de produits d'hygiène, d'un téléviseur en cellule à titre gratuit, et de la remise d'un «viatique sortant » à la libération.

À Alençon, une aide financière de 8 euros est allouée par le Secours catholique aux indigents désignés par le service de l'économat.

À Argentan, un prêt de 15 euros est consenti par l'association socioculturelle du centre de détention aux détenus indigents nouvellement arrivés. Ceux qui demeurent ensuite dans cette situation peuvent choisir entre une aide mensuelle de 23 euros ou un téléviseur gratuit et une aide vestimentaire, et bénéficient de l'envoi d'un jouet à Noël aux enfants par l'association La Cordée, et de la prise en charge des titres de transport en cas de permission de sortir ou de libération par le Secours catholique. Un «viatique sortant» leur est remis à la libération.

À la maison d'arrêt de Caen, l'indigence est reconnue en deçà de 45 euros. Un prêt de 10 euros est accordé aux arrivants par l'association socioculturelle de l'établissement, ainsi que le don de tabac, de timbres et de vêtements. Une aide financière mensuelle de 17 euros est ensuite apportée par le Secours catholique.

La remise d'un «viatique sortant» a lieu; dans les situations les plus précaires la prise en charge du titre de transport peut se produire.

Au centre pénitentiaire, la réflexion menée au cours de l'année 2003 allait dans le sens d'établir une feuille nominative d'indigence, de fournir gratuitement des vêtements, de mettre en ouvre un dispositif de bourses d'études, de remettre une enveloppe de dépannage aux détenus libérés du SMPR.

d. Les activités socio-éducatives, culturelles et sportives

Sélectionnées et pilotées par les SPIP, elles reçoivent un soutien conséquent des associations socio-éducatives, culturelles et sportives animées au sein de chaque établissement pénitentiaire.

Dépassant le degré d'une simple vertu occupationnelle, ces activités tiennent une place importante dans le quotidien carcéral et sont un vecteur fort d'intégration sociale.

Leur développement et leur diversification au sein des détentions demeurent néanmoins tributaires, là encore, de l'espace que peuvent laisser les architectures carcérales.

Cette réalité laisse très vite entrevoir le manque d'espace dont pâtissent les maisons d'arrêt de la région, contrairement aux deux établissements pour peines.

Chaque établissement de la région offre néanmoins, avec les moyens matériels dont il dispose, des activités aux détenus.
Ainsi, à titre d'exemple, durant les années 2001 et 2002, à la maison d'arrêt de Caen, quelques hommes majeurs ont pu s'adonner à la lecture, participer à un atelier d'écriture, à l'élaboration d'un journal, à des cours de littérature, de musique, d'arts plastiques, de théâtre, d'informatique; des séances de football et de musculation leur ont également été proposées régulièrement.

Les mineurs ont eu accès à un atelier d'écriture et à des cours de judo et de tennis de table, en plus des activités sportives traditionnelles en milieu carcéral.

Les femmes ont pu prendre part, quant à elles, à des cours de couture, de danse contemporaine et de stretching, et ont pu bénéficier d'une initiation à l'informatique.

Diversifiées, ces activités n'ont toutefois empli, dans la semaine, que quelques heures du vécu carcéral, et n'ont concerné que des groupes de petit effectif.

À l'inverse, les condamnés du centre pénitentiaire de Caen ont quotidiennement et aisément accès aux nombreuses activités qu'autorise un bâtiment dédié au déroulement de spectacles et de nombreux clubs: musique, théâtre, arts plastiques, informatique, maquettisme, bricolage, poterie, jeux de société, vidéo, journal interne...

Les infrastructures sportives offrent également de belles possibilités (trois grandes cours de promenade, un vaste terrain de sports et un grand gymnase, trois lieux réservés à la musculation), et permettent de fréquentes rencontres avec des équipes sportives extérieures.

\section{Les dispositifs d'insertion économique et professionnelle}

Ils reposent sur les trois piliers complémentaires que sont l'enseignement général, la formation professionnelle et le travail pénitentiaire.

Toutefois, il importe de cerner que l'intention de travailler, à des fins de rémunération ou d'occupation du temps, l'emporte fréquemment, en prison, sur le souhait de participer à une formation qualifiante ou à un enseignement lié aux savoirs de base.

\section{a. Le travail pénitentiaire}

Les formes de travail pénitentiaire observées dans la région sont majoritairement celles du service général, du travail de production en concession pour le compte d'entreprises privées ou pour celui d'associations.

Le Service général pourvoit à l'emploi d'une partie de la population carcérale au sein de chacune des structures pénitentiaires de la région.

En effet, il procure en moyenne une dizaine de postes de travail au sein des trois petites maisons d'arrêt, et offre une quarantaine d'emplois à la maison d'arrêt de Caen ; il constitue également une source de revenus pour plus d'une soixantaine de condamnés du centre pénitentiaire de Caen et pour plus de quatre-vingts détenus à Argentan.

Rémunérés par l'Administration pénitentiaire, ces emplois s'avèrent souvent peu attrayants pour la population pénale au sein des établissements où d'autres formes de travail pénitentiaire peuvent assurer un revenu plus élevé. 
Cette réalité s'observe en effet dans les structures caennaises et à Argentan.

À l'inverse, les places au Service général attirent plus volontiers les détenus incarcérés à Coutances, Cherbourg et Alençon où elles représentent les possibilités principales, voire exclusives, de travail.

Il importe de souligner néanmoins que les missions confiées aux auxiliaires du Service général n'offrent, en elles-mêmes et en l'état, aucune qualification ou savoirfaire apte à s'inscrire dans le projet de réinsertion socioprofessionnelle d'un détenu.

De ce fait, un point mérite d'être fait sur certains efforts consentis ou escomptés au centre de détention d'Argentan et à la maison d'arrêt de Caen.

À Argentan, des liens existent entre le Service général, la formation professionnelle et la dynamique d'insertion. Les détenus classés peuvent appuyer leur pratique professionnelle sur la consultation de fiches de poste et le renseignement de grilles d'évaluation.

Puis quelques-uns d'entre eux ont pu participer, au cours de l'année 2002, à une action «adaptation à l'emploi » liée au montage et au démontage de pièces d'ordinateur, destinée à aboutir à une certification de compétence.

Parallèlement à cela, le Service général, terrain d'intervention de l'entreprise privée ELYO, chargée de la maintenance, a encadré la période d'application en entreprise de six stagiaires de la formation professionnelle.

À Caen, l'intention a été affichée, au cours de ces derniers mois, d'intégrer, dans une démarche qualifiante, l'observation de la norme HACCP (analyse des dangers, points critiques pour leur maîtrise en matière de sécurité alimentaire) dans les pratiques liées à la distribution des repas et au nettoyage des locaux communs.

Le travail en concession pour le compte d'entreprises privées ou d'associations est également développé au sein de certaines détentions de Basse-Normandie; il ne requiert, et ne confere souvent que peu de qualifications particulières.

Son développement est infime ou très fluctuant dans les trois petites maisons d'arrêt où l'espace manque pour agencer des lieux de travail et de stockage.

Les établissements de la Manche n’hébergent aucun atelier de production; deux postes de travail permettent à Cherbourg de couvrir des livres pour la bibliothèque municipale, et deux autres assuraient un revenu aux femmes incarcérées à Coutances, réalisant des travaux de blanchisserie pour une société de services.

À Alençon, des tâches de pliage, de collage, de façonnage occupent, de façon assez irrégulière dans le temps, une quinzaine de détenus entre les murs d'un petit atelier situé au troisième étage de l'édifice.

La situation est tout autre à la maison d'arrêt de Caen : l'établissement possède en effet deux ateliers de travail en concession, installés au rez-de-chaussée de la "détention hommes", ainsi qu'une salle dédiée à cette activité au « quartier femmes».

Au cours de l'année 2001, le travail en concession a fourni un emploi à une moyenne mensuelle de 45 personnes ( 63 en février, 26 en septembre).
Trois donneurs d'ordres différents ont ainsi conduit la population pénale masculine et majeure à réaliser du paillage de chaises en cellule, des filets et des travaux de conditionnement, de pliage, de collage, en atelier.

Un quatrième concessionnaire $\mathrm{a}$, quant à lui, permis à quelques femmes de confectionner des «bijoux fantaisie».

La situation au centre pénitentiaire de Caen a connu d'importantes mutations au cours de l'année 2002, avec le départ d'un donneur d'ordres historique qui employait près de $45 \%$ des quelque 200 condamnés classés sur la vaste zone des ateliers. Cette zone a d'ailleurs fait l'objet d'une étude de restructuration et de modernisation.

À la fin de l'année 2002, 5 entreprises donnaient du travail aux détenus, consistant en des tâches :

- de façonnage et de conditionnement;

- de traitement de palettes et de déchets;

- de mécanique générale, usinage, montage de pièces de vélomoteur;

- de montage de circuits électroniques.

Par ailleurs, 4 associations procuraient une activité à une quinzaine de condamnés dans les domaines liés à l'imprimerie, la conception de logiciels, l'audiovisuel, et la restauration collective.

Pour finir, à Argentan, le travail en concession fourni à la population pénale découle des choix effectués par le prestataire privé GEPSA, dans le cadre du marché de fonctionnement des établissements à gestion mixte (2002/2009).

Ainsi, sur une zone d'ateliers de $1875 \mathrm{~m}^{2}$, en 2002, l'établissement a été le lieu de conditionnement de DVD, d'ordinateurs, d'assemblage et de montage d'ensembles en plastique, entre autres travaux. Jusqu'en avril 2002, la société SAGEM a apporté des châssis de téléphone-fax à monter, ce qui témoignait d'un savoir-faire élevé et d'un souci de qualité valorisant l'image des ateliers.

Le nombre moyen des actifs relevés, en 2002, dans le cadre d'une activité de production a été de 101.

\section{b. Les formations professionnelles}

Le développement des formations professionnelles en détention repose sur les choix effectués par les SPIP et les partenariats pouvant se nouer avec certains acteurs locaux tel le Conseil régional.

Alors qu'aucune formation professionnelle n'existe pour l'heure dans les maisons d'arrêt de Caen et d'Alençon, celles de Coutances et de Cherbourg sont respectivement le terrain d'une initiation au maquettisme industriel, et d'une formation aux métiers du bâtiment second œuvre.

Organisées en plusieurs sessions annuelles, dans un petit atelier en détention, ces formations s'adressent à des groupes de 12 stagiaires.

L'offre de formation professionnelle développée au centre pénitentiaire de Caen découle d'une concertation réalisée entre différents partenaires: SPIP du Calvados, direction de l'établissement, DDTEFP, AFPA, ANPE, Éducation nationale...

Les discussions menées en 2003 ont permis de valider la poursuite de la préparation au Certificat de formation 
professionnelle Menuiserie-Ébénisterie ouverte à 16 stagiaires (10 reçus sur 16 participants au CFP menuiserie, 6 reçus au CFP ébénisterie en 2002) et la pérennisation de la préparation de 12 stagiaires au Certificat d'aptitude professionnelle agricole de travaux paysagers ( 6 reçus sur 10 participants en $2001 / 2002$ ).

Le projet de mettre en place une préparatoire sectorielle aux formations de technicien d'industrie à l'intention d'une dizaine de détenus a par ailleurs été étudié.

Offrant une cinquantaine de places, quatre formations professionnelles préqualifiantes ou qualifiantes ont été proposées aux détenus du centre de détention d'Argentan, en 2002, dans les domaines suivants :

- CFP Magasinier / Gestionnaire de stock;

- préqualification aux métiers du second œuvre du Bâtiment;

- préqualification aux métiers de l'horticulture, des jardins et espaces verts;

- module «Élaboration de projet, techniques de recherche d'emploi ».

\section{c. L'enseignement général et la lutte contre l'illettrisme}

L'enseignement général et la lutte contre l'illettrisme sont assurés au sein des six détentions de la région, soit dans le cadre de cours collectifs ou individuels, soit dans le cadre d'une formation à distance.

Une réponse, à tous les niveaux et besoins de connaissance, peut être fournie par des enseignants de l'Éducation nationale, attachés à une unité locale de l'enseignement.

Le repérage des situations d'illettrisme s'opère de manière systématique, dans le cadre d'audiences réalisées à l'arrivée des détenus en milieu carcéral.

Les trois petites maisons d'arrêt de la région bénéficient de l'intervention d'un unique enseignant titulaire, assisté de deux collègues vacataires, et ont à disposition dans leurs locaux une unique salle de classe.

La maison d'arrêt de Caen dispose de deux salles de classe au « quartier hommes », et d'une salle au « quartier mineurs » et au « quartier femmes».

Outre ces moyens matériels plus développés, elle fait intervenir 5 enseignants titulaires et 3 autres vacataires, ainsi que 3 assistants de formation employés dans le cadre des «emplois jeunes».

D'autres acteurs interviennent également dans ce champ: étudiants du Génépi, enseignants du GRETA, visiteurs de prison...

Le centre pénitentiaire de Caen dispose de moyens humains et matériels (4 salles de classe, locaux d'audience) assez similaires à ceux de la maison d'arrêt, les deux structures étant rattachées à une seule et même unité locale de l'enseignement.

À Argentan, l'enseignement général proposé aux condamnés est le fait de 3 enseignants titulaires, et de 4 vacataires. Il importe de mentionner l'intervention de l'atelier de formation de base "La maison des mots", dans le champ de la lutte contre l'illettrisme.

Il convient de souligner, par ailleurs, que l'enseignement constitue, au sein des deux établissements pour peines de la région, une activité largement concurrencée par les opportunités de travail ou de formation professionnelle.

Enfin, des activités périphériques et complémentaires de l'enseignement sont proposées aux détenus des trois plus grands établissements pénitentiaires de BasseNormandie: ateliers de lecture, d'écriture, initiation à l'informatique, code de la route, cours de navigation et permis hauturier...

\section{Orientation, suivi et préparation à la sortie des détenus en Basse-Normandie}

\section{a. Accueil et suivi des publics en détention}

Ils s'opèrent, au sein des maisons d'arrêt, à travers les différents moments que constituent les audiences et entretiens individuels, les commissions de classement à une activité (travail, formation professionnelle, enseignement...), et les commissions d'application des peines.

Chacun de ces moments d'échange, en présence ou en l'absence du détenu, fait intervenir différents acteurs du milieu carcéral : personnels de direction et de surveillance, travailleurs sociaux, responsables du travail pénitentiaire, de la formation professionnelle, de l'enseignement, juge d'application des peines...

Une réunion d'observation pluridisciplinaire particulière existe par ailleurs à la maison d'arrêt de Caen et concerne les mineurs incarcérés.

Une fois par mois, différents professionnels se rassemblent dans les locaux extérieurs du Service médicopsychologique régional, situés à quelques pas de l'établisement, et évoquent tour à tour la situation personnelle de chaque mineur: membres du SMPR et du service médical, membre de la direction de la maison d'arrêt, gradé de détention, surveillant pénitentiaire et travailleur social « référents-mineurs », éducateurs de la Protection judiciaire de la jeunesse.

$\mathrm{Au}$ sein des deux établissements pour peines, les condamnés font l'objet d'un accueil et d'un suivi en détention, assez analogues.

Un accueil collectif, puis individuel leur est assuré afin qu'ils connaissent rapidement les possibilités offertes sur chaque site en termes de travail, de formation générale et professionnelle, d'activités socio-éducatives, culturelles et sportives...

À Argentan, les condamnés rencontrent un acteur institutionnel supplémentaire à leur arrivée : le Service Emploi Formation.

Placé sous la responsabilité du prestataire privé, ce service assure ainsi deux phases importantes à l'arrivée des condamnés : «l'accueil, information et mobilisation » et «le bilan, évaluation, orientation ».

Les commissions pluridisciplinaires de classement et les commissions d'application des peines assurent, comme en maison d'arrêt, une individualisation des peines privatives de liberté.

Elles s'inscrivent néanmoins dans le cadre et la philosophie du Projet d'exécution de peine (PEP).

Ce dispositif, animé par un psychologue référent, incite le condamné à devenir acteur de sa peine en s'impliquant dans un projet librement consenti et contractualisé : 
démarche thérapeutique, formation, amélioration du comportement, indemnisation des victimes...

Une fois par an, les observations rassemblées par les acteurs principaux du milieu carcéral, et consignées dans le livret individuel du détenu, donnent lieu à une évaluation des progrès réalisés ou des résistances affichées par l'intéressé.

\section{b. Préparation à la sortie, accès aux droits sociaux et aménagements de peine}

Le retour des individus dans la société, à l'issue d'une période d'incarcération, peut s'opérer de manière directe ou progressive.

Il doit cependant être préparé au mieux afin de lutter contre les risques de récidives.

Aussi, au sein des petites maisons d'arrêt de la région, différents acteurs de l'insertion sociale et professionnelle extérieurs au milieu carcéral assurent-ils des permanences en détention afin d'orienter ou accompagner les condamnés arrivant en fin de peine : représentants del'ANPE, des missions locales, des PAIO (Permanences d'accueil, d'information et d'orientation), de l'AFPA, de l'ASSEDIC, de la CPAM.

Un dispositif de préparation à la sortie, plus structuré, «le Module d'orientation approfondie », existait à la maison d'arrêt de Caen jusqu'à la fin de l'année 2002, cofinancé par l'Administration pénitentiaire et l'ANPE.

S'adressant à un public dont le reliquat de peine était inférieur à 6 mois, il faisait intervenir le GRETA puis l'ANPE ou la Mission locale afin d'élaborer avec le détenu un projet de réinsertion, en partenariat avec d'autres partenaires extérieurs.

Supprimée au sein de L'ANPE depuis le début de l'année 2003, cette mesure est aujourd'hui remplacée par une nouvelle prestation: l'Objectif projet en groupe et individuel.

Destiné à préparer les détenus à sortir du milieu carcéral avec un projet de réinsertion, ce dispositif pose le pro-

blème de son financement dans le long terme, étant donné que l'ANPE n'y participe plus et contraint le SPIP du Calvados à puiser dans son budget de fonctionnement.

Les établissements pour peines de Basse-Normandie possèdent également un dispositif de préparation à la sortie.

Outre l'intervention bimensuelle d'un conseiller de l'ANPE, les condamnés du quartier « centre de détention » du centre pénitentiaire de Caen peuvent solliciter leur intégration au DIDAF (Dispositif d'insertion des détenus par l'accompagnement de la formation).

Mis en œuvre par le GRETA, cet outil permet aux participants de préparer leur projet de sortie à l'aide d'ateliers visant l'évaluation des acquis, l'analyse des compétences requises au regard des projets individuels, la recherche documentaire, l'information sur les droits sociaux et les mesures pour l'emploi, la technique de recherche d'emploi...

De la même manière à Argentan, le PIDO (Point d'information, de diagnostic et d'orientation) constitue pour les détenus, au cœur de la détention, un lieu d'accueil et de rencontre avec les partenaires institutionnels et associatifs extérieurs : ANPE, Mission locale, AFPA, associations engagées dans la lutte contre l'alcoolisme et les toxicomanies, foyers d'hébergement...

Une coordinatrice sociale chargée de l'animation et du suivi du dispositif assure le lien avec les services de l'établissement pénitentiaire, et veille à ce qu'un «dossier $\mathrm{PIDO}$ » et des fiches d'entretien soient constitués pour chaque condamné ayant consulté un partenaire extérieur.

Ces documents, accessibles aux travailleurs sociaux, permettent de suivre l'évolution du projet de chaque individu, et de l'évoquer auprès du juge dans le cadre des commissions d'application des peines.

En dernier lieu, il importe de préciser que les condamnés privés de liberté peuvent bénéficier d'aménagements de peine, et quitter partiellement ou totalement le milieu carcéral par anticipation.

Chaque année, des mesures de suspension ou de fractionnement de peine, des placements à l'extérieur, avec ou sans surveillance continue du personnel pénitentiaire, des placements en semi-liberté ou sous surveillance électronique, ainsi que des libérations conditionnelles sont octroyés aux condamnés présentant des gages sérieux de réinsertion socioprofessionnelle.

\section{Propositions émises par le Conseil économique et social régional}

Dans le cadre de la réflexion qu'il a menée, le CESR de Basse-Normandie a jugé pertinent de dégager cinq types de propositions parmi lesquels figure la nécessité de soutenir le développement et la pérennisation des dispositifs d'insertion au sein des détentions bas-normandes.

Ici ou là en effet, les conditions d'exercice du maintien des liens familiaux peuvent être améliorées, en matière de transport, d'hébergement ou d'accueil des familles venant en visite au parloir.

Cette difficulté vaut également pour certains condamnés quittant temporairement le milieu carcéral dans le cadre des permissions de sortir.

Puis, au sein de certains sites, limités par la rigidité de leur architecture, le développement des activités socioculturelles et sportives, ainsi que les échanges possibles avec le monde extérieur doivent être encouragés.

Enfin, si dans la région un développement et une répartition satisfaisants de la démarche de repérage et de lutte contre l'illettrisme, ainsi que de l'enseignement général existent, d'importantes évolutions sont à engager ici ou là en matière de formation professionnelle et de travail pénitentiaire.

Pour cela, la Direction régionale des services pénitentiaires de Rennes aurait peut-être à afficher plus localement ses compétences et ses capacités de production afin de procurer aux détenus des emplois professionnellement qualifiants.

Une seconde préconisation émise par le CESR souligne l'intérêt d'accompagner la prise en charge des populations les plus fragilisées en milieu carcéral.

Certains publics plus fragiles que d'autres nécessitent en effet une attention particulière au sein des détentions 
de la région: détenus indigents, handicapés, malades enclins à sortir de prison pour raison de santé.

Différentes voies de soutien en faveur de ces populations paraissent envisageables : élargissement au sein des établissements pénitentiaires des commissions de repérage et de traitement de l'indigence, allocation de bourses d'études aux plus nécessiteux, amélioration de la prise en charge matérielle des personnes handicapées, développement de l'offre d'accueil et de traitement pour les malades quittant le milieu carcéral...

En troisième point, le CESR incite à favoriser le développement des mesures offrant un retour progressif à la vie libre.

Toute personne privée de liberté doit pouvoir bénéficier, si elle l'estime utile, d'un accompagnement et d'une préparation à la sortie.

Par ailleurs, le développement des mesures d'aménagement de peine ramenant progressivement certains condamnés à la liberté doit être favorisé.

En cela, le soutien éventuel que les collectivités territoriales pourraient apporter dans la région en matière d'offre d'emplois et d'aide à l'hébergement apparaît déterminant.

Enfin, une évaluation de l'efficacité des dispositifs d'insertion internes au milieu carcéral serait utilement menée à travers le suivi de parcours empruntés, sur le territoire régional, par les publics sortis du champ de compétence de l'Administration pénitentiaire.

À côté de ces trois pistes de réflexion et d'action potentielles concernant les publics incarcérés en BasseNormandie, le CESR s'intéresse, dans une quatrième proposition, à la situation professionnelle des personnels pénitentiaires affectés dans la région.

Au sein des structures carcérales de petite taille, ces personnels apparaissent assez peu nombreux en effectifs. De ce fait, ils rencontrent de réelles difficultés à s'impli- quer dans des actions de formation continue, par manque de moments à dégager sur le temps de travail.

Par ailleurs, la pénibilité à long terme des missions assumées au contact direct et répété d'une population carcérale complexe légitimerait que les personnels pénitentiaires aient accès, en cours de carrière, à des passerelles menant à d'autres tâches professionnelles.

Pour terminer, le CESR a élevé au rang de préconisation la nécessité de mieux intégrer l'institution pénitentiaire dans les réseaux institutionnels et de partenariat locaux.

Le fonctionnement et le rôle précis de l'institution pénitentiaire sont souvent mal connus du grand public et de nombreux acteurs locaux.

De ce fait, la représentation de l'Administration pénitentiaire mériterait de se développer davantage au sein d'instances impliquées dans la prévention de la délinquance ou l'observation sociale.

Par ailleurs, la transformation des structures carcérales en Établissements publics pénitentiaires dotés d'un conseil d'administration semble aujourd'hui essentielle.

Autorisée par la loi de 1987 relative au Service public pénitentiaire et recommandée dans un rapport du Sénat, cette évolution doit permettre aux acteurs locaux de s'impliquer plus directement dans la vie des établissements pénitentiaires en siégeant à leur conseil d'administration.

Il importe de garder à l'esprit que tout individu incarcéré est susceptible de retrouver un jour la liberté; la prison ne peut, de ce fait, se résumer à une «école du crime et de la délinquance » prenant le monde extérieur comme terrain d'expérimentation des acquis.

En s'impliquant davantage dans le développement des dispositifs d'insertion en milieu carcéral, les acteurs publics, privés et associatifs de la région peuvent aider à minimiser le risque de récidive chez certains publics et sauvegarder ainsi l'équilibre de la société. 